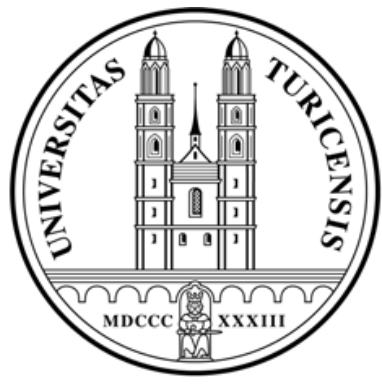

Institute for Empirical Research in Economics

University of Zurich

Working Paper Series

ISSN 1424-0459

Working Paper No. 211

Efficient elicitation of utility and probability weighting functions

Pavlo Blavatskyy

November 2004 


\title{
Efficient elicitation of utility and probability weighting functions
}

\author{
Pavlo Blavatskyy* \\ Institute for Empirical Research in Economics \\ University of Zurich \\ Winterthurerstrasse 30 \\ CH-8006 Zurich \\ Switzerland \\ Phone: $+41(1) 6343586$ \\ Fax: $+41(1) 6344978$ \\ e-mail: pavlo.blavatskyy@iew.unizh.ch
}

November 2004

\begin{abstract}
:
Elicitation methods in decision making under risk allow a researcher to infer the subjective utilities of outcomes as well as the subjective weights of probabilities from the observed preferences of an individual. An optimally efficient elicitation method is proposed, which takes into account the inevitable distortion of preferences by random errors and minimizes the effect of such errors on the inferred utility and probability weighting functions. Under mild assumptions, the optimally efficient method for eliciting utilities (weights) of many outcomes (probabilities) is the following three-stage procedure. First, a probability is elicited whose subjective weight is one half. Second, an individual's utility function is elicited through the midpoint chaining certainty equivalent method employing the probability elicited at the first stage as an input. Finally, an individual's probability weighting function is elicited through the probability equivalent method.
\end{abstract}

Keywords: decision theory, rank-dependent expected utility, cumulative prospect theory, von Neumann-Morgenstern utility, probability weighting, elicitation

JEL Classification codes: C91, D81

\footnotetext{
* I am grateful to Christian Ewerhart, Peter Wakker, and the participants of FUR XI conference (Paris, June 2004) for numerous helpful comments on the previous drafts of this paper. Part of this paper was written while I was on a study stay at the School of Economics, University of Nottingham, whose hospitality is acknowledged. Ganna Pogrebna helped a lot with English copy editing.
} 


\section{Efficient elicitation of utility and probability weighting functions}

\section{Introduction}

A choice-based elicitation procedure allows to infer the economic characteristics of an individual from the observed choice decisions of this individual. In choice under risk (e.g. Knight 1921) an important characteristic of an individual is his or her utility of outcomes (e.g. von Neumann and Morgenstern 1944). One of the first attempts to elicit an individual's utility function $u: \mathrm{R} \rightarrow \mathrm{R}$ can be found in Ramsey (1931). Classical non-parametric elicitation methods such as certainty equivalent and probability equivalent methods (e.g. Farquhar 1984) allow a researcher to infer the utility function of an individual without assuming a specific parametric form and without excluding the heterogeneity in the individual's preferences. Unfortunately, the classical elicitation methods are not robust when individuals distort or misperceive probabilities (e.g. Karmarkar 1978, McCord and de Neufville 1986, von Winterfeldt and Edwards 1986, Tversky and Fox 1995).

Prominent descriptive decision theories such as rank-dependent expected utility theory, or RDEU, (e.g. Quiggin 1982) and cumulative prospect theory, or CPT, (e.g. Tversky and Kahneman 1992) model the probability distortions in decision making under risk through a probability weighting function $w:[0,1] \rightarrow[0,1]$. The method for eliciting subjective utility function is called robust if the inferred utility function of an individual is independent from the probability weighting function of an individual. The tradeoff method, the first robust elicitation method, was proposed by Wakker and Deneffe (1996). Based on the tradeoff method, Abdellaoui (2000) generated a robust non-parametric elicitation of both utility and probability weighting functions. Other robust though not completely non-parametric elicitation methods were proposed by Gonzalez and Wu (1999) and Bleichrodt and Pinto (2000). However, the existing literature on 
the elicitation methods does not address the role of random error in decision making under risk. This paper constructs a robust non-parametric procedure for eliciting an individual's utility and probability weighting functions which is also optimally efficient i.e. it minimizes the propagation of random errors ${ }^{1}$.

Camerer (1989), Starmer and Sugden (1989) and Wu (1994) provide an extensive experimental evidence that there is some degree of randomness in the observed individual preference between lotteries. Smith and Walker (1993) and Harless and Camerer (1994, p.1265) found that real incentives reduce the randomness in individual responses. However, a researcher eliciting an individual's utility and probability weighting functions typically cannot afford the use of real incentives (e.g. Fennema and van Assen, 1998, Abdellaoui, 2000, Bleichrodt and Pinto, 2000, Abdellaoui et al. 2003, Etchart-Vincent 2004). Thus, the design of the elicitation methods should address fundamental problems caused by random error. An efficient elicitation procedure minimizes the impact of random errors on the inferred utility and probability weighting functions of an individual. To make a formal argument the structure of the stochastic element must be specified.

There is no consensus in the literature on the structure of stochastic utility. Harless and Camerer (1994, pp. 1260-1261) propose a constant choice-independent error rate with which the true asymmetric preference relation of an individual can reverse. Such a model does not work particularly well in the context of elicitation methods since these mathods rely on the symmetric (indifference) preference relation of an individual. Luce and Suppes (1965, p.335) and Camerer and Ho (1994, p.187) propose a logit form of stochastic utility. Loomes and Sugden $(1995,1998)$ argue that random errors directly affect a preference relation of an individual rather than an

\footnotetext{
${ }^{1}$ Abdellaoui et al. (2004) referred to an elicitation method as efficient in a different sense-when an elicitation method is composed of fewer measurement questions. Following Wakker and Deneffe (1996) I will call such a method as less laborious.
} 
individual's utility of lotteries. Hey and Orme (1994, p.1301) and Gonzalez and Wu (1999) propose an additive, normally distributed error term on the utility scale. Primarily for simplicity reasons and as a reasonably justifiable assumption, this paper also employs an additive, independently distributed error term on the RDEU-scale. With this structure of an error term, an elicitation procedure is claimed to be optimally efficient if it minimizes the expected sum of squared errors of the inferred utilities (weights) of a fixed number of elicited outcomes (probabilities).

The elicitation methods considered in this paper allow a researcher to infer the values of utility and probability weighing functions of an individual from the elicited subjective indifference relations between the lotteries. This paper considers two-outcome lotteries only. The notation $(x, p, y)$ denotes a lottery that yields an outcome $x$ with probability $p$ and an outcome $y<x$ with probability $1-p$. In the remainder of this paper it is assumed that the appropriate technique for extracting the indifference relation of an individual between two lotteries is available (see section 5.2 in Farquhar 1984 for a review). The indifference relations to be elicited are interrelated so that they impose sufficient restrictions on the utility and probability weighting functions of an individual. This enables a researcher to infer the values of the latter: an individual is indifferent between the lotteries $L_{1}$ and $L_{2}$ if and only if his or her utility from the lottery $L_{1}$ is equal to his or her utility from the lottery $L_{2}$ plus a random error term. According to the expected utility theory, utility from the lottery $(x, p, y)$ is given by $p \cdot u(x)+(1-p) \cdot u(y)$, while both RDEU and CPT (if $x$ and $y$ are gains) suggest that this utility equals to $w(p) \cdot u(x)+(1-w(p)) \cdot u(y)$. 
The remainder of this paper is organized as follows. Section two reviews the existing elicitation methods. Section three introduces a three-stage procedure which is proposed to optimize the efficiency of a robust non-parametric elicitation. Section four concludes.

\section{Existing elicitation methods}

This section reviews the literature on several elicitation methods, frequently sited in the paper. Readers, familiar with the existing literature on the elicitation methods, can skip this section without the loss of continuity.

\subsection{Certainty equivalence method}

Certainty equivalent (CE) method allows to elicit a sure outcome $C E(L)$, which is called a certainty equivalent, such that an individual is indifferent between $C E(L)$ for certain and a lottery L (e.g. Farquhar 1984). Using the fractile CE method, a researcher elicits the von Neumann-Morgenstern utility function of an individual by obtaining a sequence of certainty equivalents $C E\left(L_{i}\right)$ for the lotteries $L_{i}\left(x, p_{i}, y\right)$ where $[y, x]$ is an interval on which utility function is elicited and $p_{i}$ is a sequence of probabilities (e.g. von Neumann-Morgenstern 1944). ${ }^{2}$ According to the deterministic expected utility theory, an individual's utility of outcome $C E\left(L_{i}\right)$ is just $p_{i}$ when $u(y)=0$ and $u(x)=1$ are normalized by assumption (e.g. Keeney and Raiffa 1976).

Using the chaining CE method, a researcher picks several probabilities $p_{i}$ and elicits first the certainty equivalents $C E\left(L_{i}\right)$ of the lotteries $L_{i}\left(x, p_{i}, y\right)$. Subsequently, the researcher elicits the certainty equivalents of the lotteries $L_{1 i}\left(x, p_{i}, C E\left(L_{1}\right)\right), \quad L_{2 i}\left(C E\left(L_{1}\right), p_{i}, C E\left(L_{2}\right)\right), \ldots$

\footnotetext{
${ }^{2}$ Unless otherwise indicated, throughout the paper index $i$ goes from unity to some natural number $n \geq 1$ i.e. $i \in[1, n]$.
} 
$L_{i i}\left(C E\left(L_{i}\right), p_{i}, y\right)$ and so forth (e.g. Farquhar 1984). The midpoint chaining refers to a special case of the chaining CE method, when only one probability $p_{1}=0.5$ is used (e.g. Krzysztofowicz and Duckstein 1980). The CE method remains valid only under the expected utility theory and it is not robust to the non-linear weighting of probabilities. Different biases and distortions associated with the CE method were discovered inter alia by Krzysztofowicz and Duckstein (1980), Hershey et al. (1982), and Hershey. and Schoemaker (1985). The CE method is a non-parametric elicitation method.

\subsection{Probability equivalence method}

The probability equivalent (PE) method allows to elicit a probability $p_{i}$ such that an individual is indifferent between the lottery $L_{i}\left(x, p_{i}, y\right)$ and an outcome $z_{i} \in[y, x]$ for certain. According to the deterministic expected utility theory, an individual's utility of $z_{i}$ is just $p_{i}$ when $u(y)=0$ and $u(x)=1$ are normalized by assumption (e.g. Farquhar 1984). The PE method allows to elicit the von Neumann-Morgenstern utility function of an individual without chaining the earlier responses to the lotteries involved in subsequent comparisons. However, many individuals find the probability judgment to be cognitively demanding (e.g. Karmarkar 1978). The PE method remains valid only under the expected utility theory and it is not robust to the non-linear probability weighting. Hershey and Schoemaker (1985) provide an extensive experimental evidence of systematic discrepancies between utility functions elicited through the CE and PE methods. The PE method is a non-parametric elicitation method.

\subsection{Tradeoff method}

The tradeoff (TO) method proposed by Wakker and Deneffe (1996) is a robust elicitation method i.e. it allows to elicit the von Neumann-Morgenstern utility function of an individual that is not affected by the non-linear probability weighting. The TO method allows to elicit a 
sequence of outcomes $x_{1}, \ldots, x_{n}$, which is called a standard outcome sequence, such that an individual is indifferent between lotteries $\left(x_{i-1}, p, R\right)$ and $\left(x_{i}, p, r\right)$ (e.g. Wakker and Deneffe 1996, Fennema and van Assen 1998). The reference outcomes $0 \leq r<R<x_{0}$ are free parameters chosen by a researcher.

The probability $p$ is also arbitrary chosen by a researcher. Wakker and Deneffe (1996) recommend to choose $p=1 / 3$ because a typical individual is most probable to have a minimum subjective distortion of probability in the neighborhood of $p=1 / 3$ (e.g. Tversky and Fox 1995, Prelec 1998). Apparently, this consideration motivated Wakker and Daneffe (1996), Fennema and van Assen (1998), Abdellaoui et al. $(2002,2004)$ and Etchart-Vincent (2004) to use the TO method with $p=1 / 3$. Other probabilities used in the literature are $p=1 / 2$ (e.g. Bleichrodt and Pinto 2000) and $p=2 / 3$ (e.g. Abdellaoui 2000).

According to the deterministic expected utility theory, RDEU, and CPT an individual's utility of $x_{i}$ is just $i / n$ when $u(y)=0$ and $u(x)=1$ are normalized by assumption (e.g. Wakker and Deneffe 1996). ${ }^{3}$ Thus, the elements of a standard outcome sequence are equally spaced in terms of the subjective utility. The TO method remains valid when the probabilities are weighted non-linearly or even when the probabilities are unknown. Thus, the TO method is applicable both to the decisions under risk and uncertainty. The TO method is a non-parametric elicitation method.

\subsection{Elicitation of probability weighting function}

CE, PE and TO methods described above allow to elicit the von Neumann-Morgenstern utility function of an individual. However, to describe an individual's decision making under risk

\footnotetext{
${ }^{3}$ For simplicity, this paper considers only lotteries yielding gains (monetary outcomes above a reference point, typically zero). Thus, the predictions of rank-dependent expected utility theory and cumulative prospect theory coincide. The extension of TO method to losses is straightforward (e.g. Etchart-Vincent 2004)
} 
completely his or her probability weighting function must be elicited as well. This section summarizes two approaches for eliciting a probability weighting function that are based on the TO method. Thus, as a starting point, the TO method is used to elicit a standard outcome sequence $x_{1}, \ldots, x_{n}$.

Bleichrodt and Pinto (2000) propose the following approach to elicit a subjective probability weighting function. For any probability $p \leq 0.5$ a researcher elicits an outcome $x^{\prime}$ such that an individual is indifferent between the lottery $\left(x_{i}, p, x_{j}\right)$ and the lottery $\left(x_{k}, p, x^{\prime}\right)$, where outcomes $x_{i}, x_{j}, x_{k}$ are the elements of the standard outcome sequence such that $x_{k} \geq x_{i} \geq x_{j}$. According to the deterministic RDEU and CPT, $w(p)=\frac{j-n \cdot u\left(x^{\prime}\right)}{j+k-i-n \cdot u\left(x^{\prime}\right)}(e . g$. equation (8) in Bleichrodt and Pinto 2000). For any probability $p>0.5$ a researcher elicits an outcome $x^{\prime}$ such that an individual is indifferent between the lottery $\left(x_{i}, p, x_{j}\right)$ and the lottery $\left(x^{\prime}, p, x_{k}\right)$, where outcomes $x_{i}, x_{j}, x_{k}$ are the elements of the standard outcome sequence such that $x_{i} \geq x_{j} \geq x_{k}$. According to the deterministic RDEU and CPT, $w(p)=\frac{j-k}{n \cdot u\left(x^{\prime}\right)+j-k-i}$ (e.g. equation (9) in Bleichrodt and Pinto 2000).

To infer the weight of a probability $p$ a researcher must know the utility of an outcome $x^{\prime}$. Since in general the outcome $x^{\prime}$ does not belong to the standard outcome sequence, Bleichrodt and Pinto (2000) calculate $u\left(x^{\prime}\right)$ through a linear interpolation $u\left(x^{\prime}\right) \approx \frac{l}{n}+\frac{x^{\prime}-x_{l}}{\left(x_{m}-x_{l}\right) n}$ where an outcome $x_{l}$ is the element of the standard outcome sequence such that $x_{l} \leq x^{\prime} \leq x_{l}+1$. Thus, the approach of Bleichrodt and Pinto (2000) is based on the parametric fitting of a piecewise linear utility function. 
Abdellaoui (2000) proposes a different approach. A subjective probability weighting function is elicited through the PE method using the standard outcome sequence as an input. A researcher elicits a sequence of probabilities $p_{1}, \ldots, p_{n-1}$, which is called a standard sequence of probabilities, such that an individual is indifferent between the lottery $\left(x_{n}, p_{i}, x_{0}\right)$ and an outcome $x_{i}$ for certain. According to the deterministic RDEU and CPT, $w\left(p_{i}\right)=i / n$ even without assuming a normalization $u\left(x_{0}\right)=0, u\left(x_{n}\right)=1$. Notice that according to the deterministic expected utility theory a probability $p_{i}$ is uniquely determined as $p_{i}=i / n$ and, thus, its elicitation is nothing but a consistency check.

The approach of Abdellaoui (2000) changes the response scale i.e. an individual first gives his or her answers in terms of monetary outcomes (in the TO questions) and then - in terms of probabilities (in the PE questions). Tversky et al. (1988) and Delquie (1993) provide an evidence that a preference elicitation may be distorted when the response scale is varied. The approach of Abdellaoui (2000) is non-parametric.

\subsection{Other elicitation methods}

Gonzalez and $\mathrm{Wu}$ (1999) propose a robust method for the simultaneous elicitation of utility and probability weighting functions through the alternating least squares approach. To infer the subjective utilities of outcomes $x_{1}, \ldots, x_{n}$ and the subjective weights of probabilities $p_{1}, \ldots, p_{m}$, a researcher experimentally elicits the certainty equivalents $C E_{i j l}$ of (many) lotteries $\left(x_{i}, p_{j}, x_{l}\right), i, l \in[1, n], j \in[1, m]$. Subsequently, $u\left(x_{i}\right)$ and $w\left(p_{j}\right)$ are chosen as free parameters. The subjective utility of every certainty equivalent $u\left(C E_{i j l}\right)$ is approximated through a linear interpolation of the utility function based on the assumed values of $u\left(x_{i}\right)$. Finally, the parameters $u\left(x_{i}\right)$ and $w\left(p_{j}\right)$ are chosen iteratively to minimize the discrepancy between $u\left(C E_{i j l}\right)$ and the 
utility of a corresponding lottery $\left(x_{i}, p_{j}, x_{l}\right)$ under RDEU and CPT. The approach of Gonzalez and $\mathrm{Wu}(1999)$ is based on the parametric fitting of a piecewise linear utility function.

Abdellaoui et al. (2004) propose a robust two-step procedure for eliciting an individual's utility function. First, Abdellaoui et al. (2004) elicit a probability $p$ whose subjective weight is one half. This elicitation is done through the approach of Abdellaoui (2000), described in section 2.4, with the standard outcome sequence consisting of three outcomes. Second, Abdellaoui et al. (2004) elicit an individual's utility function through the midpoint chaining CE method described in section 2.1 with the elicited probability $p=w^{-1}(0.5)$ being used instead of the probability $p_{1}=0.5$. The approach of Abdellaoui et al. (2004) is non-parametric.

Ghirardato et al. (2003) proposed a theoretical method for the robust elicitation of an individual's utility function. An outcome $z \in[y, x]$ is called a preference average of outcomes $x$ and $y$ given a non-degenerate probability $p$ if an individual is indifferent between the lottery $(x, p, y)$ and the compound lottery $((x, p, z), p,(z, p, y))$. According to the RDEU, $u(z)=0.5 \cdot u(x)+0.5 \cdot u(y)($ e.g. Ghirardato et al. 2003). Furthermore, a preference average of outcomes $x$ and $z$ has an utility of $0.75 \cdot u(x)+0.25 \cdot u(y)$. Proceeding along these lines, a researcher is able to infer an individual's utility function over the entire interval $[y, x]$ through the chaining elicitation of preference averages. However, Abdellaoui et al. (2004) argue that the experimental elicitation of preference averages is a cognitively demanding task for the subjects because the method of Ghirardato et al. (2003) involves compound lotteries. The method of Ghirardato et al. (2003) is non-parametric. 


\section{Three-stage procedure}

To infer an individual's utility of $n$ outcomes and an individual's weight of $n$ probabilities, a researcher employs a three-stage $(3 \mathrm{~S})$ procedure, which is non-parametric and robust to the non-linear weighting of probabilities. First, a researcher chooses a relatively small number $k \in[1, n-1]$ of non-degenerate probabilities whose weights are elicited through the approach of Abdellaoui (2000) described in section 2.4. At one extreme, when a researcher chooses $k=n-1$ the proposed $3 \mathrm{~S}$ procedure consists of only one stage and coincides with the approach of Abdellaoui (2000). At the other extreme, when a researcher chooses $k=1$ the first stage of $3 \mathrm{~S}$ procedure coincides with the first stage of the method of Abdellaoui et al. (2004) as described in section 2.5 .

Second, a researcher elicits an individual's utility function through the chaining CE method described in section 2.1. $k$ probabilities elicited at the first stage are used as an input for the CE method. The subjective weight of the probabilities elicited at the first stage is already known. Thus, a researcher can control for the non-linear weighting of probabilities and the chaining CE method becomes robust to probability distortions. After $m \in N$ iterations of the chaining CE method a researcher obtains $(k+1)^{m+1}$ outcomes at which the value of an individual's utility function is known. For simplicity reasons I assume that $n=(k+1)^{m+1}$ so that exactly $m$ iterations of the chaining CE method are required to elicit the desired number of outcomes. When $k=1$ the first and the second stage of $3 \mathrm{~S}$ procedure coincide with the method of Abdellaoui et al. (2004).

Third, a researcher elicits an individual's probability weighting function through the PE method described in section 2.2. The outcomes elicited at the second stage are used as an input for the PE method. Since TO, CE and PE methods are non-parametric elicitation methods, the 
proposed $3 \mathrm{~S}$ procedure, which is a combination of $\mathrm{TO}, \mathrm{CE}$ and $\mathrm{PE}$ methods, is also a nonparametric elicitation procedure. The $3 \mathrm{~S}$ procedure can be further extended in the following way. Consider the situation when $n<<(k+1)^{m+1}$. After the third stage a researcher proceeds again with the second stage, using a richer set of probabilities whose subjective weight has been just elicited at the third stage. Thus, the second and the third stages are repeated in a cycle until a desired number $n$ of outcomes/probabilities is elicited. This extension, however, is beyond the scope of this paper.

The choice parameter for a researcher is a number $k$ of non-degenerate probabilities whose subjective weights are elicited at the first stage. For a fixed number $n$ of outcomes (probabilities) whose subjective utility (weight) is to be elicited: the number of iterations $m$ is uniquely determined from the choice of $k$ e.g. $m=\log _{k+1} n-1$. In the following sub-sections 3.13.3 the propagation of random errors is analyzed for every stage of the proposed $3 \mathrm{~S}$ procedure. Sub-section 3.4 demonstrates how a researcher chooses the optimal number $k$ to minimize the propagation of error across all stages of the elicitation procedure.

\subsection{Propagation of error at stage one}

At the first stage a researcher elicits $k \in N$ non-degenerate probabilities whose subjective weight is inferred through the approach of Abdellaoui (2000). A standard outcome sequence $x_{1}, \ldots, x_{k+1}$ is elicited initially though the TO method (presented in section 2.3). An individual is asked $k+1$ times to reveal such an outcome $x_{i}$ that makes him or her indifferent between the lotteries $\left(x_{i-1}, p, R\right)$ and $\left(x_{i}, p, r\right)$ with the reference outcomes $0 \leq r<R<x_{0}$ and a probability $p$ being fixed by a researcher. Assuming an additive, independently distributed error term $\varepsilon^{T O} \sim\left(0, \sigma_{1}^{2}\right)$ on the utility scale (e.g. Hey and Orme 1994, Gonzalez and $\left.\mathrm{Wu} 1999\right)$, the 
indifference relations elicited through the TO method can be rewritten under RDEU as well as under CPT as the system of equations (1).

$$
w(p) u\left(x_{i-1}\right)+(1-w(p)) u(R)=w(p) u\left(x_{i}\right)+(1-w(p)) u(r)+\varepsilon_{i}^{T O}, i \in[1, k+1]
$$

Assuming a normalization $u\left(x_{0}\right)=0, u\left(x_{k+1}\right)=1$, the recursive system (1) can be rewritten as (2).

$$
u\left(x_{i}\right)=\frac{i}{k+1}+\frac{1}{w(p)}\left(\sum_{j=1}^{i} \varepsilon_{j}^{T O}-\frac{i}{k+1} \sum_{j=1}^{k+1} \varepsilon_{j}^{T O}\right), i \in[1, k+1]
$$

It follows from the system of equations (2) that an individual's utility of every elicited outcome $\quad x_{i}$ has the expectation $E\left(u\left(x_{i}\right)\right)=\frac{i}{k+1}$ and the variance $\operatorname{var}\left(u\left(x_{i}\right)\right)=\frac{\sigma_{1}^{2}}{w^{2}(p)} i \cdot\left(1-\frac{i}{k+1}\right)$. Thus, the utility of the median member of the standard outcome sequence $u\left(x_{(k+1) / 2}\right)$ has the highest variance $\frac{(k+1) \sigma_{1}^{2}}{4 w^{2}(p)}$ and the utility of the first and the last member of the standard outcome sequence have the lowest variance. In other words, under the TO method random errors distort the inferred utility of the medium members of the standard outcome sequence more severely. The propagation of an error is less severe for the first and the last members of the standard outcome sequence. In contrast, Wakker and Deneffe (1996, p. 1148) intuitively suggested that the error propagation is increasing for the later members of the standard outcome sequence.

Subsequently, a researcher elicits a standard sequence of probabilities $p_{1}, \ldots, p_{k}$ through the PE method (presented in section 2.2) using the elicited standard outcome sequence $x_{1}, \ldots, x_{k+1}$. Assuming an additive, independently distributed error term $\varepsilon^{P E} \sim\left(0, \sigma_{2}^{2}\right)$ on the utility scale, the indifference relations elicited through the PE method can be rewritten under RDEU as well as under CPT as a system of equations (3). 


$$
w\left(p_{i}\right)=u\left(x_{i}\right)+\varepsilon_{i}^{P E}=\frac{i}{k+1}+\frac{1}{w(p)}\left(\sum_{j=1}^{i} \varepsilon_{j}^{T O}-\frac{i}{k+1} \sum_{j=1}^{k+1} \varepsilon_{j}^{T O}\right)+\varepsilon_{i}^{P E}, i \in[1, k]
$$

In general, a random error occurring when the PE method is used can have a different variance than the random error occurring under the TO method because two methods employ different types of elicitation questions. Thus, $\sigma_{2}>\sigma_{1}$ when the questions employed in the PE method are more cognitively demanding than the questions employed in the TO method and $\sigma_{2}<\sigma_{1}$ otherwise. It follows from the system of equations (3) that an individual's weight of every elicited probability $p_{i}$ has the expectation $E\left(w\left(p_{i}\right)\right)=\frac{i}{k+1}$ and the variance $\operatorname{var}\left(w\left(p_{i}\right)\right)=\frac{\sigma_{1}^{2}}{w^{2}(p)} i \cdot\left(1-\frac{i}{k+1}\right)+\sigma_{2}^{2}$. Under the PE method random errors do not propagatethe error $\varepsilon_{i}^{P E}$ affects solely the inferred weight of the probability $p_{i}$ and it does not affect the inferred weights of the other probabilities.

\subsection{Propagation of error at stage two}

At the end of the first stage a standard outcome sequence $x_{1}, \ldots, x_{k+1}$ is already elicited though the TO method. An individual's utility of $k+1$ members of this sequence is determined by the system of equations (2). At the second stage a researcher elicits a more refined grid of outcomes whose subjective utility is inferred through the chaining CE method. The standard outcome sequence partitions the interval $\left[x_{0}, x_{k+1}\right]$ into $k+1$ smaller intervals. For every smaller interval a researcher elicits the certainty equivalents of $k$ probability mixtures of its upper and lower bound. The probability mixtures are constructed using a standard sequence of probabilities elicited at the first stage. Overall a researcher elicits $k(k+1)$ certainty equivalents during the first iteration of the chaining CE method. These newly elicited certainty equivalents together with the 
standard outcome sequence partition the interval $\left[x_{0}, x_{k+1}\right]$ into $(k+1)^{2}$ smaller intervals. During the second iteration of the chaining CE method, a researcher elicits the certainty equivalents of $k$ probability mixtures of the upper and the lower bound of each of those $(k+1)^{2}$ intervals. The same procedure is iterated for $m$ times. Figure 1 illustrates the construction of the refined grid of outcomes.

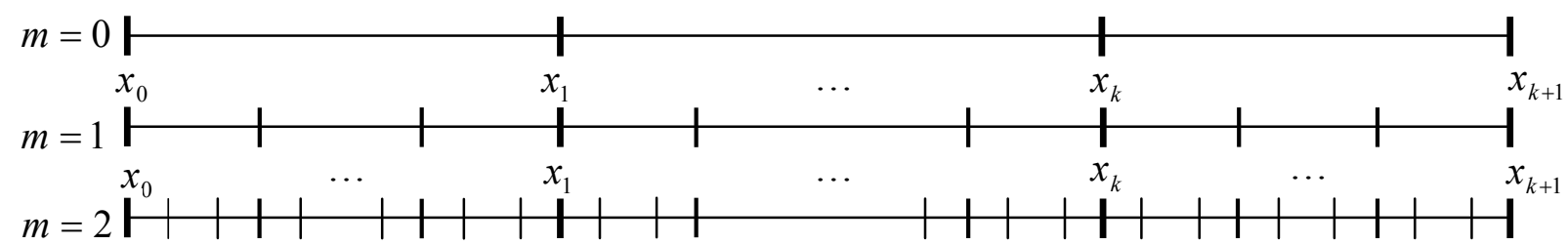

Figure 1 Chaining CE method with multiple probabilities

During an iteration $\mu \in[1, m]$ of the chaining CE method a researcher elicits $k(k+1)^{\mu}$ certainty equivalents. Thus, by the end of the second stage a researcher has elicited $(k+1)^{m+1}$ outcomes overall whose subjective utility can be inferred in a robust non-parametric way. To facilitate a subsequent formal argument, let $y_{0}, \ldots, y_{(k+1)^{m+1}}$ denote the sequence of outcomes elicited by the end of the second stage and numbered consecutively so that $y_{i}>y_{j}$ if and only if $i>j$. Let $a_{m} a_{m-1} \ldots a_{0}$ denote a natural number written in a number system with a base value $k+1$. Obviously, every digit $a_{i}$ of such number must be $a_{i} \in[0, k]$ for any $i \in[0, m]$. The conversion of the number $a_{m} a_{m-1} \ldots a_{0}$ into a decimal number system is $a_{m}(k+1)^{m}+a_{m-1}(k+1)^{m-1}+\ldots+a_{1}(k+1)+a_{0}$. With the above notation, every outcome elicited by the end of the second stage, except for the highest outcome $y_{(k+1)^{m+1}}=x_{k+1}$, can be denoted as $y_{a_{m} a_{m-1} \ldots a_{0}}, a_{i} \in[0, k], i \in[0, m]$. In particular, the members of the standard outcome sequence

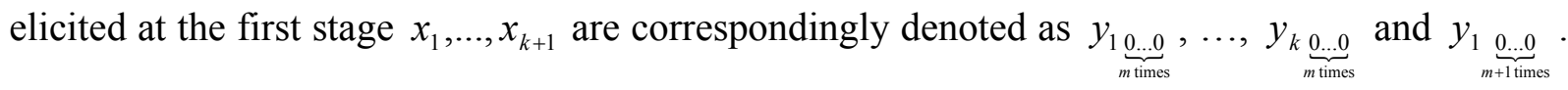


The indifference relations elicited during an iteration $\mu \in[1, m]$ of the chaining CE method can be then formally written as (4).

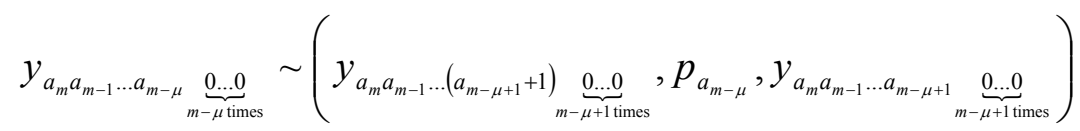

$$
\begin{aligned}
& \forall a_{m-\mu}, a_{m-\mu+1}, \ldots, a_{m} \in[0, k]
\end{aligned}
$$

Notice that a number $a_{m} a_{m-1} \ldots\left(a_{m-\mu+1}+1\right) \underset{m-\mu+1 \text { times }}{0 \ldots .0}$ becomes a number $a_{m} a_{m-1} \ldots\left(a_{m-\mu+2}+1\right) \underset{m-\mu+2 \text { times }}{0 \ldots 0}$ when $a_{m-\mu+1}=k$, a number $a_{m} a_{m-1} \ldots\left(a_{m-\mu+3}+1\right) \underset{m-\mu+3 \text { times }}{0 \ldots 0}$ when $a_{m-\mu+1}=a_{m-\mu+2}=k, \ldots$ and a number $1 \underbrace{0 \ldots 0}_{m+1 \text { times }}$ when $a_{m-\mu+1}=\ldots=a_{m}=k$. Assuming an additive, independently distributed error term $\varepsilon^{C E} \sim\left(0, \sigma_{3}^{2}\right)$ on the utility scale, the indifference relations (4) can be rewritten under RDEU as well as CPT as a system of equations (5).

$$
\begin{aligned}
& u\left(y_{a_{m} a_{m-1} \ldots a_{m-\mu}} \underset{m-\mu \text { times }}{0.0}\right)=u\left(y_{a_{m} a_{m-1} \ldots a_{m-\mu+1}} \underset{m-\mu+1 \text { times }}{0 \ldots 0}\right)+w\left(p_{a_{m-\mu}}\right) \times \\
& \times\left(u\left(y_{a_{m} a_{m-1} \ldots\left(a_{m-\mu+1}+1\right)} \underset{m-\mu+1 \text { limes }}{0 \ldots 0}\right)-u\left(y_{a_{m} a_{m-1} \ldots a_{m-\mu+1}}^{0} \underset{m-\mu+1 \text { times }}{0 \ldots 0}\right)\right)+\varepsilon_{a_{m} a_{m-1} \ldots a_{m-\mu}}^{C E} \underset{m-\mu \text { times }}{0 \ldots .0} \\
& \forall a_{m-\mu}, a_{m-\mu+1}, \ldots, a_{m} \in[0, k], \mu \in[1, m]
\end{aligned}
$$

It follows from the recursive system (5) that random errors propagate in a non-linear manner at the second stage. To infer the subjective utility of a newly elicited certainty equivalent, a researcher multiplies the utilities of previously elicited outcomes (affected by random errors) by the weight elicited at the first stage (which is also affected by random errors). The non-linear propagation of errors creates an addition problem for a researcher. To make the unbiased inferences of an individual's utility function, a researcher needs to estimate the variances $\sigma_{1}^{2}, \sigma_{2}^{2}$ and $\sigma_{3}^{2}$. In general, under the non-linear propagation of errors the expected values of an individual's utility function are not equally spaced at the outcomes elicited through the chaining 
CE method. To simplify the formal analysis it is assumed in what follows that random errors propagate linearly at stage two. Specifically, instead of system (5) a recursive system (6) is used.

$$
\begin{aligned}
& u\left(y_{a_{m} a_{m-1} \ldots a_{m-\mu}} \underset{m-\mu \text { times }}{0.0 .0}\right)=u\left(y_{a_{m} a_{m-1} \ldots a_{m-\mu+1}} \underset{m-\mu+1 \text { titimes }}{0.0}\right)+w\left(p_{a_{m-\mu}}\right) \times \\
& \times\left(E\left(u\left(y_{a_{m} a_{m-1} \ldots\left(a_{m-\mu+1}+1\right)} \underset{m-\mu+1 \text { times }}{0 \ldots 0}\right)\right)-E\left(u(y_{a_{m} a_{m-1} \ldots a_{m-\mu+1}}^{0} \underbrace{0 \ldots 0}_{m-\mu+1 \text { times }})\right)\right)+\varepsilon_{a_{m} a_{m-1} \ldots a_{m-\mu}}^{C E} \underset{m-\mu \text { times }}{0 \ldots 0} \\
& \forall a_{m-\mu}, a_{m-\mu+1}, \ldots, a_{m} \in[0, k], \mu \in[1, m]
\end{aligned}
$$

Proposition 1 For any outcome $y_{a_{m} a_{m-1} \ldots a_{0}}$ elicited by the end of the second stage, the inferred subjective utility of $y_{a_{m} a_{m-1} \ldots a_{0}}$ has an expectation of $E\left(u\left(y_{a_{m} a_{m-1} \ldots a_{0}}\right)\right)=\frac{a_{m} a_{m-1} \ldots a_{0}}{(k+1)^{m+1}}$.

Proof of proposition 1 and all subsequent propositions is presented in appendix.

It follows from proposition 1 that at the first and the second stages a researcher elicits a sequence of outcomes that are expected to be equally spaced in terms of subjective utility. Specifically, after an iteration $\mu$ of the chaining CE method the elicited sequence of outcomes divides an interval $\left[y_{0}, \ldots, y_{1} \underset{m+1 \text { times }}{0.0}\right]=\left[x_{0}, x_{k+1}\right]$ into $(k+1)^{\mu+1}$ smaller intervals. The expected length of each of these smaller intervals is $1 /(k+1)^{\mu+1}$ in terms of subjective utility. By the end of the second stage a researcher completes the task of utility function elicitation. At the third stage only a refined probability weighting function is elicited.

\subsection{Propagation of error at stage three}

At the end of the first stage a standard sequence of probabilities $p_{1}, \ldots, p_{k}$ is already elicited. The subjective weights of $p_{1}, \ldots, p_{k}$ can be inferred from equation (3) as $1 /(k+1), \ldots, k /(k+1)$ correspondingly. At the third stage the PE method described in section 2.2 is used to elicit a more refined grid of probabilities whose subjective weights can be inferred by a 
researcher. Specifically, for every outcome $y_{a_{m} a_{m-1} \ldots a_{0}}$ elicited at the second stage, an individual is asked to reveal such a probability $q_{a_{m} a_{m-1} \ldots a_{0}}$ that makes him or her indifferent between the lottery $\left(x_{k+1}, q_{a_{m} a_{m-1} \ldots a_{0}}, x_{0}\right)$ and the outcome $y_{a_{m} a_{m-1} \ldots a_{0}}$ for certain. Assuming an additive and independently distributed error term $\varepsilon^{P E} \sim\left(0, \sigma_{2}^{2}\right)$ on the RDEU-scale, the indifference relations elicited at the third stage can be formally written as the system of equations (7).

$$
\begin{gathered}
w\left(q_{a_{m} a_{m-1} \ldots a_{0}}\right)=u\left(y_{a_{m} a_{m-1} \ldots a_{0}}\right)+\varepsilon_{a_{m} a_{m-1} \ldots a_{0}}^{P E} \\
\forall a_{m}, a_{m-1}, \ldots, a_{0} \in[0, k]
\end{gathered}
$$

It follows from (7) that $E\left(w\left(q_{a_{m} a_{m-1} \ldots a_{0}}\right)\right)=E\left(u\left(y_{a_{m} a_{m-1} \ldots a_{0}}\right)\right)=\frac{a_{m} a_{m-1} \ldots a_{0}}{(k+1)^{m+1}}$ with the later equality due to proposition 1. The system of equations (7) also implies that random errors do not propagate at the third stage - an error $\varepsilon_{a_{m} a_{m-1} \ldots a_{0}}^{P E}$ affects only the elicitation of the probability $q_{a_{m} a_{m-1} \ldots a_{0}}$ and it does not affect the elicitation of the other probabilities. By the end of the third stage a researcher elicits $n=(k+1)^{m+1}$ probabilities that are expected to be equally spaced in terms of subjective weight.

\subsection{Optimally efficient elicitation procedure}

Let us assess the efficiency of the $3 \mathrm{~S}$ procedure with respect to the elicitation of an individual's utility function. The first step is to calculate the expected sum of squared errors affecting the inferred utilities of $n$ elicited outcomes.

Proposition 2 The expected sum of squared errors of the inferred utilities of $n=(k+1)^{m+1}$ outcomes elicited through the $3 \mathrm{~S}$ procedure is given by equation (8) when $k \neq 1$ and by equation (9) when $k=1$. 


$$
\begin{gathered}
S=\left[\frac{k+1}{6}\left(n-\frac{1}{n}\right)+\right. \\
\left.\frac{(k+1)^{2}}{2\left(k^{2}+k-1\right)}\left(\frac{k^{3}\left(k^{\log _{k+1} n-1}-1\right)}{(k-1)^{2}}+\frac{n-k-1}{n k}\right)-\frac{\left(\log _{k+1} n-1\right)(k+1)^{2}}{2(k-1)}\right] \frac{\sigma_{1}^{2}}{w^{2}(p)}+ \\
+\left[\log _{k+1} n-1-\frac{n-k-1}{n k}\right] \sigma_{2}^{2}+\left(\log _{k+1} n-1\right) n \frac{k}{k+1} \sigma_{3}^{2} \\
\left.S\right|_{k=1}=\left[\frac{n}{3}-\frac{13}{3 n}+\left(\log _{2} n\right)^{2}-3 \log _{2} n+4\right] \frac{\sigma_{1}^{2}}{w^{2}(p)}+\left[\log _{2} n-2+\frac{2}{n}\right] \sigma_{2}^{2}+\left(\log _{2} n-1\right) \frac{n}{2} \sigma_{3}^{2}
\end{gathered}
$$

The optimally efficient $3 \mathrm{~S}$ procedure aims to minimize the expected sum of squared errors (8)-(9). To design the optimally efficient elicitation procedure a researcher chooses the number $k \in[1, n-1]$ that minimizes the right hand side (RHS) of the equations (8)-(9). The first part of the RHS of equation (8) increases in $k$. However, the second and the third part of the RHS of equation (8), which are presented together in the second line of the equation, both decrease in $k$. Thus, when $\sigma_{1}^{2}=0$ the first part of the RHS of equation (8) cancels out and the optimal $3 \mathrm{~S}$ procedure is to set $k$ to its maximum $k=n-1$. In other words, when the TO elicitation questions are cognitively undemanding i.e. in the TO questions the individuals report their indifference relations without random errors; the optimal elicitation procedure is the simple TO method as described in section 2.3 .

When $\sigma_{2}^{2}=\sigma_{3}^{2}=0$, the second and the third part of the RHS of equation (8) cancel out. The remaining first part of the RHS of equation (8) increases in $k$. Thus, the optimal $3 \mathrm{~S}$ procedure is to set $k$ to its minimum $k=1$. In other words, when the PE and CE elicitation questions are cognitively undemanding i.e. they induce no random errors in the elicited responses of an individual; the optimal elicitation procedure is the method of Abdellaoui et al. (2004) as described in section 2.5. More general result is obtained in proposition 3.

Proposition 3 Assuming an additive and independently distributed error term on the RDEU-scale, such that an error is most distorting in the TO questions and least distorting -in the 
CE questions i.e. $\sigma_{1}^{2} \geq \sigma_{2}^{2} \geq \sigma_{3}^{2}$; there is a number $n^{*}$ such that the optimally efficient procedure for eliciting an individual's utility function of $n \geq n^{*}$ outcomes is the $3 \mathrm{~S}$ procedure with $k=1$ (the method of Abdellaoui et al. 2004).

There is an established empirical support for the assumption that $\sigma_{1}^{2} \geq \sigma_{2}^{2} \geq \sigma_{3}^{2}$. Wakker and Deneffe (1996) report that in the experiments the untrained individuals find the TO elicitation questions harder to understand compared to the PE and CE elicitation questions. A plausible explanation is that the TO questions are more cognitively demanding because they involve a comparison of two lotteries rather than a comparison of one lottery and an outcome for certain as in the PE and CE questions. This finding can be interpreted that random errors have a more distorting effect in the TO elicitation questions compared to the PE and CE questions. In other words, $\sigma_{1}^{2} \geq \sigma_{2}^{2}$ and $\sigma_{1}^{2} \geq \sigma_{3}^{2}$. Ronen (1973) and Karmarkar (1978) report that the untrained individuals find the PE elicitation questions more difficult that the $\mathrm{CE}$ questions. $\mathrm{A}$ plausible explanation is that the PE method requires an individual to make a probability judgment — a task that occurs seldom in real life experiences. Therefore, the untrained individuals are not accustomed to make a probability judgment and they find it more difficult. This observation can be interpreted that random errors have a more distorting effect in the PE elicitation questions compared to the $\mathrm{CE}$ questions. To summarize, the empirical evidence suggests that an untrained individual is characterized by an inequality $\sigma_{1}^{2} \geq \sigma_{2}^{2} \geq \sigma_{3}^{2}$.

A threshold $n^{*}$ from proposition 3 depends on the volatilities $\sigma_{1}^{2}, \sigma_{2}^{2}, \sigma_{3}^{2}$ and the subjective weight $w(p)$ of the probability $p$ used by a researcher in the TO method at the first stage of the $3 \mathrm{~S}$ procedure. Specifically, the higher the volatility $\sigma_{1}^{2}$ of random errors in the TO elicitation questions, the lower the volatilities $\sigma_{2}^{2}$ and $\sigma_{3}^{2}$ of random errors in the PE and CE 
elicitation questions, and the lower the weight $w(p)$, the lower is the threshold $n^{*}$. Thus, if the 3S procedure with $k=1$ is optimally efficient for eliciting $n \geq n^{*}$ outcomes when $\sigma_{1}^{2}=\sigma_{2}^{2}=\sigma_{3}^{2}$ then it is moreover optimally efficient for eliciting $n \geq n^{*}$ outcomes when $\sigma_{1}^{2} \geq \sigma_{2}^{2} \geq \sigma_{3}^{2}$. When $\sigma_{1}^{2}=\sigma_{2}^{2}=\sigma_{3}^{2}$ i.e. the distorting effect of random errors is the same in TO, PE and CE elicitation questions, a (conservative) value of the threshold $n^{*}$ is $n^{*}=11$ if $w(p) \leq 1 / 3, n^{*}=19$ if $w(p) \leq 1 / 2$ and $n^{*}=64$ if $w(p) \leq 2 / 3$. As discussed in section 2.3 the most popular probability chosen for the TO method is $p=1 / 3$, which typically has a subjective weight $w(p)=1 / 3$. Thus, the practical implication of the main theoretical result of this paper is that the optimally efficient method for eliciting utilities of eleven or more outcomes is the $3 \mathrm{~S}$ procedure with $k=1$ (under mild assumptions of proposition 3). No definite statement can be made on the efficiency of elicitation methods for eliciting utilities of less than eleven outcomes, unless some stronger assumptions are imposed.

The existing experimental studies that elicit the utility function of an individual by means of the simple TO method construct a standard outcome sequence with $n \leq 6$ outcomes. Wakker and Deneffe (1996) and Fennema and van Assen (1998) use $n=4$. Etchart-Vincent (2004) uses $n=5$. Bleichrodt and Pinto (2000), Abdellaoui (2000) and Abdellaoui et al. (2002, 2003) use $n=6$. Von Winterfeldt and Edwards (1986, p. 254) intuitively recommend to use $3 \leq n \leq 6$. The only experimental study that employs the $3 \mathrm{~S}$ procedure with $k=1$, Abdellaoui et al. (2004), elicits a subjective utility of $n=11$ losses and $n=8$ gains. Thus, a current experimental practice and the intuitive recommendations in the literature are consistent with the optimally efficient elicitation procedure formally derived in proposition 3 that minimizes the expected sum of squared errors. 
So far the optimally efficient elicitation procedure was considered only for the utility function. As a next step, consider the optimally efficient elicitation procedure for the probability weighting function. It follows from the equations (3) and (7) that the expected sum of squared errors of the inferred weights of $n$ probabilities elicited through the $3 \mathrm{~S}$ procedure is just the expected sum of squared errors (8)-(9) of the inferred utilities of $n$ outcomes elicited through the 3S procedure plus a constant $n \sigma_{2}^{2}$. Since the objective function (8) to be minimized is the same in both cases (except for a constant), the optimally efficient elicitation procedure for the probability weighting function is the same as that for the utility function.

\section{Conclusion}

Propagation of random errors constitutes a fundamental challenge for the elicitation methods in decision making under risk. Although this problem was recognized since long, the existing literature is limited to the informal arguments (e.g. Wakker and Deneffe 1996) and intuitive recommendations (e.g. von Winterfeldt and Edwards 1986) how to minimize the propagation or random errors. Partially this is due to the lack of consensus on the structure of stochastic utility since little is known how random errors enter into an individual's decision making under risk. As a starting point for a formal argument, this paper assumes a specific error term — an additive, independently distributed error on the RDEU-scale — and derives an optimally efficient elicitation procedure that minimizes the expected sum of squared errors. An interesting extension of this paper is to compare its result with optimally efficient procedures derived from other structures of random error.

Under mild assumptions, the derived optimally efficient method for eliciting subjective utilities (weights) of many outcomes (probabilities) is the following three-stage procedure. First, a probability, whose subjective weight is one half, is elicited through the approach of Abdellaoui 
(2000). Second, an individual's utility function is elicited through the midpoint chaining CE method using the probability elicited at the first stage as an input. Third, an individual's probability weighting function is elicited through the PE method using the outcomes elicited at the second stage as an input. This elicitation procedure is non-parametric (no assumption about the functional form of utility and probability weighting functions is made) and robust (the inferred subjective utility function is independent from the inferred subjective probability weighting function). The first two steps of this procedure are used by Abdellaoui et al. (2004) for the experimental elicitation of utility function. Thus, this paper can be regarded as a theoretical complement of Abdellaoui et al. (2004) providing insights on the optimal efficiency of their elicitation method.

\section{References}

Abdellaoui, M. (2000) "Parameter-Free Elicitation of Utility and Probability Weighting Functions" Management Science 46, 1497-1512

Abdellaoui, M., Barrios, C. and Wakker, P. (2002) "Reconciling introspective utility with revealed preference: experimental arguments based on prospect theory" CREED, Department of Economics, University of Amsterdam

Abdellaoui, M., Bleichrodt, H. and Paraschiv, C. (2004) "Measuring loss aversion under prospect theory: a parameter-free approach“ GRID, ESTP \& ENSAM working paper

Abdellaoui, M., Vossmann, F. and Weber, M. (2003) "Choice-based Elicitation and Decomposition of Decision Weights for Gains and Losses under Uncertainty" CEPR working paper

Bleichrodt, H., J. L. Pinto (2000) “A Parameter-Free Elicitation of the Probability Weighting Function in Medical Decision Analysis" Management Science 46, 1485-1496 
Camerer, C. (1989) “An experimental test of several generalized utility theories.” Journal of Risk and Uncertainty 2, 61-104

Camerer, C. and Ho, T. (1994). "Violations of the Betweenness Axiom and Nonlinearity in Probability" Journal of Risk and Uncertainty 8, 167-196

Delquié, P. (1993) “Inconsistent Trade-Offs between Attributes: New Evidence in Preference Assessment Biases" Management Science 39, 1382-1395

Etchart-Vincent, N. (2004) "Is Probability Weighting Sensitive to the Magnitude of consequences? An Experimental Investigation on Losses" Journal of Risk and Uncertainty 28, 217-235

Farquhar, P. (1984) “Utility Assessment Methods” Management Science 30, 1283-1300

Fennema, H., M. van Assen (1998) "Measuring the utility of losses by means of the trade-off method" Journal of Risk and Uncertainty 17, 277-296

Gonzalez, R. abd Wu, G. (1999) „On the shape of the probability weighting function“ Cognitive Psychology 38, 129-166

Harless, D. and C. Camerer (1994) “The predictive utility of generalized expected utility theories" Econometrica 62, 1251-1289

Hershey, J., Kunreuther, H. and Schoemaker, P. (1982) "sources of bias in assessment procedures for utility functions" Management Science 28, 936-954

Hershey, J. and Schoemaker, P. (1985) "Probability versus Certainty Equivalence Methods in Utility Measurement: Are They Equivalent?" Management Science 31, 1213-1231.

Hey, J.D. and C. Orme (1994) "Investigating generalisations of expected utility theory using experimental data" Econometrica 62, 1291-1326

Kahneman, D. and Tversky, A. (1979) "Prospect Theory: An analysis of decision under risk" Econometrica 47, 263-291 
Karmarkar, U. (1978) "Subjectively Weighted Utility: A Descriptive Extension of the Expected Utility Model" Organizational Behavior and Human Performance 21, 61-72.

Keeney, R. and Raiffa, H. (1976) "Decisions with multiple objectives: preferences and value tradeoffs" Wiley, New York

Knight, F.H. (1921). Risk, uncertainty and profit. Houghton Mifflin, Boston

Krzysztofowicz, R. and Duckstein, L. (1980) "Strength of preference and risk attitude in utility measurement" Organizational Behavior and Human Performance 31, 88-113

Loomes, G. and Sugden, R. (1995) “Incorporating a stochastic element into decision theories" European Economic Review 39, 641-648

Loomes, G. and Sugden, R. (1998) “Testing different stochastic specifications of risky choice” Economica 65, 581-598

Luce, R. D., \& Suppes, P. (1965). Preference, utility, and subjective probability. In R. D. Luce, R. R. Bush \& E. Galanter (eds.), Handbook of mathematical psychology, Vol. III (pp. 249-410). Wiley, New York NY

McCord, M. and Neufville R. de (1983) "Fundamental Deficiency of Expected Utility Decision Analysis." In Simon French, R. Hartley, L.C. Thomas, \& Douglas J. White (Eds.), MultiObjective Decision Making, 279-305, Academic Press, New York.

McCord, M. and Neufville R. de (1986) " "Lottery Equivalents ": Reduction of the Certainty Effect Problem in Utility Assessment," Management Science 32, 56-60

Prelec, D. (1998) "The Probability Weighting Function", Econometrica, 66: 3, 497-527

Quiggin, J. (1982) “A theory of anticipated utility" Journal of Economic Behavior and Organization 3, 323-343

Ramsey, F. (1931) "Truth and probability" in "The foundations of mathematics and other logical essays" 156-198 Routledge and Kegan Paul, London 
Ronen, J. (1973) "Effects of some probability displays on choices" Organizational Behavior and Human Performance 9, 1-15

Smith, V. L. and J. Walker (1993) "Monetary rewards and decision cost in experimental economics" Economic Inquiry 31, 245-261.

Starmer, Ch. and Sugden, R. (1989) "Probability and juxtaposition effects: An experimental investigation of the common ratio effect." Journal of Risk and Uncertainty 2, 159-178

Tversky, A., D. Kahneman (1992) “Advances in Prospect Theory: Cumulative Representation of Uncertainty" Journal of Risk and Uncertainty 5, 297-323

Tversky, A. and Fox, C. (1995) "Weighing risk and uncertainty" Psychological Review 102, 269283

Tversky A., Sattath, S. and Slovic, P. (1988) "Contingent Weighting in Judgment and Choice" Psychological Review 95(3): 371-384

Tversky, A. and Wakker, P. (1995) "Risk attitudes and decision weights" Econometrica 63, $1255-1280$

von Neumann, J. and O. Morgenstern, 1944, Theory of games and economic behavior, Princeton, Princeton University Press

von Winterfeldt, D. and Edwards, W. (1986), "Decision Analysis and Behavioral Research." Cambridge University Press, Cambridge.

Wakker, P. P. and Deneffe, D. (1996) "Eliciting von Neumann-Morgenstern Utilities When Probabilities Are Distorted or Unknown” Management Science 42, 1131-1150

Wu, G. (1994) "An Empirical Test of Ordinal Independence" Journal of Risk and Uncertainty 9, $39-60$ 


\section{Appendix}

\section{Proof of proposition 1}

For convenient notation, let $\varsigma_{i}=\frac{1}{w(p)}\left(\sum_{j=1}^{i} \varepsilon_{j}^{T O}-\frac{i}{k+1} \sum_{j=1}^{k+1} \varepsilon_{j}^{T O}\right)$ denote an error distorting the inferred subjective utility (2) of an outcome $x_{i}$ that is a member of a standard outcome sequence elicited at stage one, $i \in[1 . k+1]$. In section 3.1 it was already established that $\xi_{i} \sim\left(0, \frac{\sigma_{1}^{2}}{w^{2}(p)} i\left(1-\frac{i}{k+1}\right)\right)$. Using the definition of $\xi_{i}$ we can rewrite (2) as (A1).

$$
u\left(y_{a_{m}} \underset{m \text { times }}{0 \ldots 0}\right)=u\left(x_{a_{m}}\right)=a_{m} /(k+1)+\xi_{a_{m}}
$$

Lemma A1 An individual's utility of an outcome $y_{a_{m} a_{m-1} \ldots a_{m-\mu}} \underset{m-\mu \text { times }}{0 \ldots 0}$ elicited during an iteration $\mu \in[1, m]$ of the chaining CE method is given by (A2).

$$
\begin{gathered}
u\left(y_{a_{m} a_{m-1} \ldots a_{m-\mu}} \underset{m-\mu \text { times }}{0 \ldots 0}\right)=\frac{a_{m} a_{m-1} \ldots a_{m-\mu}}{(k+1)^{\mu+1}}+\xi_{a_{m}}+\frac{1}{k+1} \xi_{a_{m-1}}+\ldots+\frac{1}{(k+1)^{\mu}} \xi_{a_{m-\mu}}+ \\
+\frac{1}{k+1} \varepsilon_{a_{m-1}}^{P E}+\ldots+\frac{1}{(k+1)^{\mu}} \varepsilon_{a_{m-\mu}}^{P E}+\varepsilon_{a_{m} a_{m-1}}^{C E} \underset{m-1 \text { times }}{0.0}+\ldots+\varepsilon_{a_{m} a_{m-1} \ldots a_{m-\mu}}^{C E} \underset{m-\mu \text { times }}{0 . \ldots 0}
\end{gathered}
$$

Proof by mathematical induction. When $\mu=1$ the system of equations (6) becomes (A3).

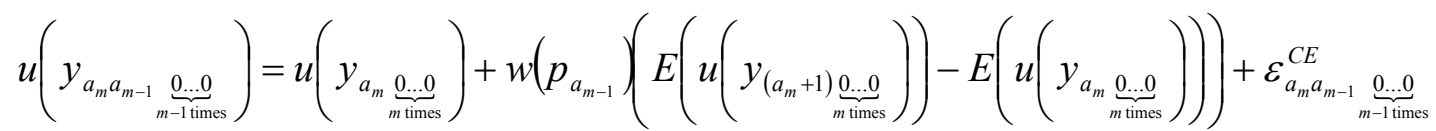

$$
\begin{aligned}
& \forall a_{m} \in[0, k], a_{m-1} \in[1, k]
\end{aligned}
$$

The outcomes $y_{a_{m} \ldots \ldots .0}^{0.0}$ and $y_{\left(a_{m}+1\right) \ldots \ldots .0}$ are members of the standard outcome sequence $x_{a_{m}}$ and $x_{a_{m}+1}$ correspondingly. An individual's utility of outcomes $y_{a_{m} \underset{m \text { times }}{0.0 .0}}$ and $y_{\left(a_{m}+1\right)} \underset{m \text { times }}{0 . .0}$ is given by 


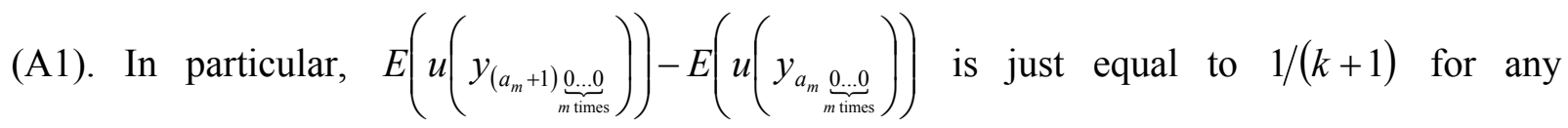
$a_{m} \in[0, k]$. Probability $p_{a_{0}}$ is elicited at stage one and its subjective weight is determined by equation (3). Thus, the system of equations (A3) can be rewritten as (A4).

$$
\begin{gathered}
u\left(y_{a_{m} a_{m-1}} \underset{m-1 \text { times }}{0.0}\right)=\frac{a_{m} a_{m-1}}{(k+1)^{2}}+\xi_{a_{m}}+\frac{1}{k+1} \xi_{a_{m-1}}+\frac{1}{k+1} \varepsilon_{a_{m-1}}^{P E}+\varepsilon_{a_{m} a_{m-1}}^{C E} \underset{m-\ldots .0}{0 . \text { times }} \\
\forall a_{m} \in[0, k], a_{m-1} \in[1, k]
\end{gathered}
$$

Thus, lemma A1 holds for $\mu=1$. Assume that lemma A1 holds also for any $\mu \in[1, m-1]$. Let us prove that lemma 1 holds for $\mu=m$ as well. When $\mu=m$ the system of equations (6) becomes (A5).

$$
u\left(y_{a_{m} \ldots a_{0}}\right)=u\left(y_{a_{m} \ldots a_{1} 0}\right)+w\left(p_{a_{0}}\right)\left(E\left(u\left(y_{a_{m} \ldots\left(a_{1}+1\right) 0}\right)\right)-E\left(u\left(y_{a_{m} \ldots a_{1} 0}\right)\right)\right)+\varepsilon_{a_{m} \ldots a_{0}}^{C E}
$$

The assumption that lemma A1 holds for any iteration $\mu \in[1, m-1]$ implies that $E\left(u\left(y_{a_{m} \ldots\left(a_{1}+1\right) 0}\right)\right)-E\left(u\left(y_{a_{m} \ldots a_{1} 0}\right)\right)=1 /(k+1)^{m}$ and $u\left(y_{a_{m} \ldots a_{1} 0}\right)=\frac{a_{m} \ldots a_{1}}{(k+1)^{m}}+\xi_{a_{m}}+\frac{1}{k+1} \xi_{a_{m-1}}+\ldots+\frac{1}{(k+1)^{m-1}} \xi_{a_{1}}+\frac{1}{k+1} \varepsilon_{a_{m-1}}^{P E}+\ldots+\frac{1}{(k+1)^{m-1}} \varepsilon_{a_{1}}^{P E}+$ $\varepsilon_{a_{m} a_{m-1}}^{C E} \underset{m-1 \text { limes }}{0.0 .}+\ldots+\varepsilon_{a_{m} \ldots a_{1} 0}^{C E}$

The weight $w\left(p_{a_{0}}\right)$ is equal to $a_{0} /(k+1)+\xi_{a_{0}}+\varepsilon_{a_{0}}^{P E}$ due to equation (3). Plugging these results into the system of equations (A5) yields (A6), which is nothing but (A2) for the case $\mu=m$.

$$
\begin{gathered}
u\left(y_{a_{m} \ldots a_{0}}\right)=\frac{a_{m} \ldots a_{0}}{(k+1)^{m+1}}+\xi_{a_{m}}+\frac{1}{k+1} \xi_{a_{m-1}}+\ldots+\frac{1}{(k+1)^{m}} \xi_{a_{0}}+\frac{1}{k+1} \varepsilon_{a_{m-1}}^{P E}+\ldots+\frac{1}{(k+1)^{m}} \varepsilon_{a_{0}}^{P E}+ \\
\varepsilon_{a_{m} a_{m-1}}^{C E} \underset{m-1 \text { times }}{0}+\ldots+\varepsilon_{a_{m} \ldots a_{0}}^{C E}
\end{gathered}
$$

Thus, lemma A1 holds also for $\mu=m$. Taking the expectation operator from the lest and the right hand side of (A6) yields immediately $E\left(u\left(y_{a_{m} a_{m-1} \ldots a_{0}}\right)\right)=\frac{a_{m} a_{m-1} \ldots a_{0}}{(k+1)^{m+1}} \cdot$ Q.E.D. 


\section{Proof of proposition 2}

The expected sum of squared errors of the inferred utilities of $n=(k+1)^{m+1}$ outcomes elicited through the $3 \mathrm{~S}$ procedure is given by (A7).

$$
S=E\left(\sum_{\mu=0}^{m} \sum_{a_{m}=0}^{k} \ldots \sum_{a_{m-\mu+1}=0}^{k} \sum_{a_{m-\mu}=1}^{k}\left(u\left(y_{a_{m} \ldots a_{m-\mu}} \underset{m-\mu \text { times }}{0 \ldots 0}\right)-\frac{a_{m} \ldots a_{m-\mu} \underset{m-\ldots \text { times }}{0}}{(k+1)^{m+1}}\right)^{2}\right)
$$

In equation (A7) we already exploited the fact that $E\left(u\left(y_{a_{m} \ldots a_{m-\mu}} \underset{m-\mu \text { times }}{0 . .00}\right)\right)=\frac{a_{m} \ldots a_{m-\mu} \underset{m-\mu \text { times }}{0}}{(k+1)^{m+1}}$ due to proposition 1. An individual's utility $u\left(y_{a_{m} \ldots a_{m-\mu}} \underset{m-\mu \text { times }}{0.0}\right)$ is determined by lemma A1 (the system of equations A2). Plugging (A2) into (A7) yields (A8).

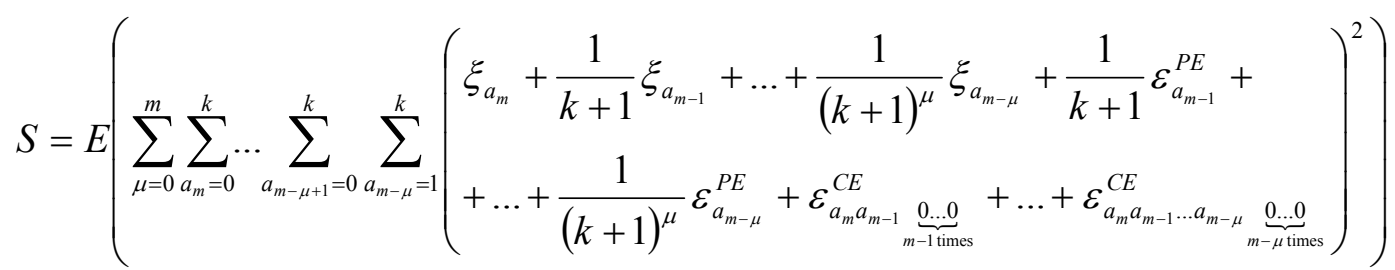

Since random errors are independently distributed one can rewrite (A8) as (A9).

$$
\begin{aligned}
& S=\sum_{\mu=0}^{m} \sum_{a_{m}=0}^{k} \ldots \sum_{a_{m-\mu+1}=0}^{k} \sum_{a_{m-\mu}=1}^{k}\left(E\left(\xi_{a_{m}}\right)^{2}+\frac{1}{(k+1)^{2}} E\left(\xi_{a_{m-1}}\right)^{2}+\ldots+\frac{1}{(k+1)^{2 \mu}} E\left(\xi_{a_{m-\mu}}\right)^{2}\right)+ \\
& +\sum_{\mu=1}^{m} \sum_{a_{m}=0}^{k} \ldots \sum_{a_{m-\mu+1}=0}^{k} \sum_{a_{m-\mu}=1}^{k}\left(\frac{2}{k+1} E\left(\xi_{a_{m}} \xi_{a_{m-1}}\right)+\frac{2}{(k+1)^{2}} E\left(\xi_{a_{m}} \xi_{a_{m-2}}\right)+\ldots+\frac{2}{(k+1)^{\mu(\mu-1)}} E\left(\xi_{a_{m-\mu}} \xi_{a_{m-\mu}}\right)\right)+ \\
& +\sum_{\mu=1}^{m} \sum_{a_{m}=0}^{k} \ldots \sum_{a_{m-\mu+1}=0}^{k} \sum_{a_{m-\mu}=1}^{k}\left(\frac{1}{(k+1)^{2}} E\left(\varepsilon_{a_{m-1}}^{P E}\right)^{2}+\ldots+\frac{1}{(k+1)^{2 \mu}} E\left(\varepsilon_{a_{m-\mu}}^{P E}\right)^{2}\right)+ \\
& +\sum_{\mu=1}^{m} \sum_{a_{m}=0}^{k} \ldots \sum_{a_{m-\mu+1}=0}^{k} \sum_{a_{m-\mu}=1}^{k}\left(E\left(\varepsilon_{a_{m} a_{m-1}}^{C E} \underset{m-1.1 \text { times }}{0.0}\right)^{2}+\ldots+E\left(\varepsilon_{a_{m} a_{m-1} \ldots a_{m-\mu}}^{C E} \underset{m-\mu \text { times }}{0.0}\right)^{2}\right)
\end{aligned}
$$

To proceed further one needs to calculate the following sums: 


$$
\begin{aligned}
\text { a) } & \sum_{\mu=0}^{m} \sum_{a_{m}=0}^{k} \ldots \sum_{a_{m-\mu+1}=0}^{k} \sum_{a_{m-\mu}=1}^{k}\left(E\left(\xi_{a_{m}}\right)^{2}+\frac{1}{(k+1)^{2}} E\left(\xi_{a_{m-1}}\right)^{2}+\ldots+\frac{1}{(k+1)^{2 \mu}} E\left(\xi_{a_{m-\mu}}\right)^{2}\right)= \\
= & \sum_{\mu=0}^{m}\left(\sum_{i=0}^{\mu-1} k(k+1)^{\mu-1-2 i}+\frac{1}{(k+1)^{\mu}}\right)\left(\sum_{b=0}^{k} E\left(\xi_{b}\right)^{2}\right)=\sum_{\mu=0}^{m}\left(\frac{(k+1)^{\mu+1}}{k+2}+\frac{1}{(k+2)(k+1)^{\mu}}\right) \frac{k(k+2)}{6} \frac{\sigma_{1}^{2}}{w^{2}(p)}= \\
= & \frac{k+1}{6}\left((k+1)^{m+1}-\frac{1}{(k+1)^{m+1}}\right) \frac{\sigma_{1}^{2}}{w^{2}(p)}=\frac{k+1}{6}\left(n-\frac{1}{n}\right) \frac{\sigma_{1}^{2}}{w^{2}(p)}
\end{aligned}
$$

where we exploited the fact that

$$
\begin{aligned}
& \sum_{b=0}^{k} E\left(\xi_{b}\right)^{2}=\sum_{b=0}^{k} \frac{\sigma_{1}^{2}}{w^{2}(p)} b \cdot\left(1-\frac{b}{k+1}\right)=\left[\frac{k(k+1)}{2}-\frac{2 k^{3}+3 k^{2}+k}{6(k+1)}\right] \frac{\sigma_{1}^{2}}{w^{2}(p)}=\frac{k(k+2)}{6} \frac{\sigma_{1}^{2}}{w^{2}(p)} \\
& \text { b) } \sum_{\mu=1}^{m} \sum_{a_{m}=0}^{k} \ldots \sum_{a_{m-\mu+1}=0}^{k} \sum_{a_{m-\mu}=1}^{k}\left(\frac{2}{k+1} E\left(\xi_{a_{m}} \xi_{a_{m-1}}\right)+\frac{2}{(k+1)^{2}} E\left(\xi_{a_{m}} \xi_{a_{m-2}}\right)+\ldots+\frac{2}{(k+1)^{\mu(\mu-1)}} E\left(\xi_{a_{m-\mu}} \xi_{a_{m-\mu}}\right)\right)= \\
& =2 \sum_{\mu=1}^{m} \sum_{i=0}^{\mu-1} \sum_{j=1}^{\mu-i} \frac{k^{i}}{(k+1)^{j}}\left(\sum_{b=0}^{k} \sum_{c=1}^{k} E\left(\xi_{b} \xi_{c}\right)\right)=\frac{k(k+1)^{2}}{2} \frac{\sigma_{1}^{2}}{w^{2}(p)} \sum_{\mu=1}^{m} \sum_{i=0}^{\mu-1} \sum_{j=1}^{\mu-i} \frac{k^{i}}{(k+1)^{j}}= \\
& =\frac{k(k+1)^{2}}{2} \frac{\sigma_{1}^{2}}{w^{2}(p)} \sum_{\mu=1}^{m} \sum_{i=0}^{\mu-1} k^{i-1}\left(1-\frac{1}{(k+1)^{\mu-i}}\right)=\frac{(k+1)^{2}}{2} \frac{\sigma_{1}^{2}}{w^{2}(p)} \sum_{\mu=1}^{m}\left(\frac{k^{\mu}-1}{k-1}-\frac{1}{(k+1)^{\mu}} \frac{k^{\mu}(k+1)^{\mu}-1}{k(k+1)-1}\right)= \\
& =\left[\frac{(k+1)^{2}}{2\left(k^{2}+k-1\right)}\left(\frac{k^{3}\left(k^{m}-1\right)}{(k-1)^{2}}+\frac{1}{k}\left(1-\frac{1}{(k+1)^{m}}\right)\right)-\frac{m(k+1)^{2}}{2(k-1)}\right] \frac{\sigma_{1}^{2}}{w^{2}(p)}
\end{aligned}
$$

where we exploited the fact that

$$
\begin{aligned}
& \sum_{b=0}^{k} \sum_{c=1}^{k} E\left(\xi_{b} \xi_{c}\right)=\sum_{b=0}^{k} \sum_{c=1}^{k} E\left(\sum_{j=1}^{b} \varepsilon_{j}^{T O}-\frac{b}{k+1} \sum_{j=1}^{k+1} \varepsilon_{j}^{T O}\right)\left(\sum_{j=1}^{c} \varepsilon_{j}^{T O}-\frac{c}{k+1} \sum_{j=1}^{k+1} \varepsilon_{j}^{T O}\right)= \\
& =\frac{\sigma_{1}^{2}}{w^{2}(p)} \sum_{b=0}^{k} \sum_{c=1}^{k}\left(\min \{b, c\}-\frac{b c}{k+1}\right)=\frac{k(k+1)^{2}}{4} \frac{\sigma_{1}^{2}}{w^{2}(p)}
\end{aligned}
$$

While calculating sum b) we implicitly assumed that $k \neq 1$ i.e. the division by $k-1$ is possible.

When $k=1$ we obtain that

b') $\sum_{\mu=1}^{m} \sum_{a_{m}=0}^{k} \ldots \sum_{a_{m-\mu+1}=0}^{k} \sum_{a_{m-\mu}=1}^{k}\left(\frac{2}{k+1} E\left(\xi_{a_{m}} \xi_{a_{m-1}}\right)+\frac{2}{(k+1)^{2}} E\left(\xi_{a_{m}} \xi_{a_{m-2}}\right)+\ldots+\frac{2}{(k+1)^{\mu(\mu-1)}} E\left(\xi_{a_{m-\mu}} \xi_{a_{m-\mu}}\right)\right)=$ 
$=2 \frac{\sigma_{1}^{2}}{w^{2}(p)} \sum_{\mu=1}^{m} \sum_{i=0}^{\mu-1}\left(1-\frac{1}{2^{\mu-i}}\right)=2 \frac{\sigma_{1}^{2}}{w^{2}(p)} \sum_{\mu=1}^{m}\left(\mu-1-\frac{1}{2^{\mu}}\right)=\left(m^{2}-m+2-\frac{1}{2^{m-1}}\right) \frac{\sigma_{1}^{2}}{w^{2}(p)}$

c) $\sum_{\mu=1}^{m} \sum_{a_{m}=0}^{k} \ldots \sum_{a_{m-\mu+1}=0}^{k} \sum_{a_{m-\mu}=1}^{k}\left(\frac{1}{(k+1)^{2}} E\left(\varepsilon_{a_{m-1}}^{P E}\right)^{2}+\ldots+\frac{1}{(k+1)^{2 \mu}} E\left(\varepsilon_{a_{m-\mu}}^{P E}\right)^{2}\right)=$

$=\sum_{\mu=1}^{m} \sum_{i=1}^{\mu} \frac{k \sigma_{2}^{2}}{(k+1)^{i}}=\sum_{\mu=1}^{m}\left(1-\frac{1}{(k+1)^{\mu}}\right) \sigma_{2}^{2}=\left[m-\frac{1}{k}\left(1-\frac{1}{(k+1)^{m}}\right)\right] \sigma_{2}^{2}=\left[m-\frac{n-k-1}{n k}\right] \sigma_{2}^{2}$

d) $\sum_{\mu=1}^{m} \sum_{a_{m}=0}^{k} \ldots \sum_{a_{m-\mu+1}=0}^{k} \sum_{a_{m-\mu}=1}^{k}\left(E\left(\varepsilon_{a_{m} a_{m-1}}^{C E} \underset{m-1 \text { times }}{0 \ldots 0}\right)^{2}+\ldots+E\left(\varepsilon_{a_{m} a_{m-1} \ldots a_{m-\mu}}^{C E} \underset{m-\mu \text { times }}{0.00}\right)^{2}\right)=m(k+1)^{m} k \sigma_{3}^{2}=m n \frac{k}{k+1} \sigma_{3}^{2}$

Plugging sums a)-d) into equation (A9) and substituting for $m=\log _{k+1} n-1$ yields immediately the RHS of equation (8). Plugging sums a), b'), c) and d) into equation (A9) and substituting for $k=1$ yields immediately the RHS of equation (9). Q.E.D.

\section{Proof of proposition 3}

The 3S procedure with $k=1$ is optimally efficient when $\Delta S=\left.S\right|_{k=2}-\left.S\right|_{k=1}>0$. The sum of squared errors $\left.S\right|_{k=2}$ is given by the equation (8) when $k=2$, and the sum of squared errors $\left.S\right|_{k=1}$ is given by the equation (9). Since $\sigma_{1}^{2} \geq \sigma_{2}^{2} \geq \sigma_{3}^{2}$, the difference in the expected sums of squared errors $\Delta S=\left.S\right|_{k=2}-\left.S\right|_{k=1}$ has a lower bound (A12).

$$
\begin{gathered}
\Delta S>\left[\frac{n}{6}+\frac{149}{60 n}-\frac{25}{4}+3 \log _{2} n-4.5 \log _{3} n-\left(\log _{2} n\right)^{2}+\frac{18}{5} 2^{\log _{3} n}\right] \frac{1}{w^{2}(p)}- \\
-\frac{n}{6}-\frac{1}{2 n}+\frac{1}{2}+\left(\frac{2 n}{3}+1\right) \log _{3} n-\left(\frac{n}{2}+1\right) \log _{2} n
\end{gathered}
$$

When $n=3$, the RHS of the equation (A12) becomes negative if the weight $w(p)$ is greater than 0.135 . Thus, the $3 \mathrm{~S}$ procedure with $k=1$ is not necessarily efficient when $n=3$. However, when $n \rightarrow+\infty$, the RHS of the equation (A12) becomes strictly positive. Since the sum of squared errors $S$ is a continuous function of $n$, there exists a threshold $n^{*} \geq 3$ such that $\Delta S>0$ for every $n \geq n^{*}$.Q.E.D. 